Meta

Journal des traducteurs

Translators' Journal

\title{
Sens et signifiance dans la traduction poétique
}

\section{Mário Laranjeira}

Volume 41, numéro 2, juin 1996

Traduction et terminologie au Brésil

Translation and Terminology in Brazil

URI : https://id.erudit.org/iderudit/003399ar

DOI : https://doi.org/10.7202/003399ar

Aller au sommaire du numéro

Éditeur(s)

Les Presses de l'Université de Montréal

ISSN

0026-0452 (imprimé)

1492-1421 (numérique)

Découvrir la revue

Citer cet article

Laranjeira, M. (1996). Sens et signifiance dans la traduction poétique. Meta, 41(2), 217-222. https://doi.org/10.7202/003399ar

\section{Résumé de l'article}

La traduction de textes pragmatiques exige du traducteur qu'il porte une attention particulière à la structure notionnelle (notions et relations notionnelles) du texte de départ de façon à rendre fidèlement la fonction communicative de celui-ci ainsi que sa tendance à !' univocité. Cependant, dans le cas de la traduction de textes poétiques, il est important de faire ressortir la prédominance du signifiant (face "matérielle" du signe) et l'obliquité sémantique constituant le passage d'accès au niveau sémiotique de signifiance et rendant possible la lecture multiple par la rupture du réfèrent externe. 


\title{
SENS ET SIGNIFIANCE DANS LA TRADUCTION POETIQUE
}

\author{
MÁrio LARANJEIRA \\ Universidade de São Paulo, São Paulo, Brésil
}

\section{Résumé}

La traduction de textes pragmatiques exige du traducteur qu'il porte une attention particulière à la structure notionnelle (notions et relations notionnelles) du texte de départ de façon à rendre fidèlement la fonction communicative de celui-ci ainsi que sa tendance $\grave{a}$ l'univocité

Cependant, dans le cas de la traduction de textes poétiques, il est important de faire ressortir la prédominance du signifiant (face «matérielle» du signe) et l'obliquité sémantique constituant le passage d'accès au niveau sémiotique de signifiance et rendant possible la lecture multiple par la rupture du référent externe.

\begin{abstract}
When translating pragmatic texts, the translator has to pay attention to the notional structure (notions and notional relations) of the source text in order to faithfully convey its communicative function, as well as its univocal tendency.

In the case of poetic translation, however, it is important that the translator bring out the prevalence of the signifier (the "material" face of the sign) and the semantic obliquity. which gives access to the semiotic level of meaning and makes multiple readings possible.
\end{abstract}

\section{Resumo}

Na tradução do texto pragmático a atenção do tradutor deve voltar-se particularmente para os conceitos a serem transmitidos e para a relação entre eles, de modo a salvaguardar a função comunicativa do texto e sua tendência à univocidade.

$\mathrm{Na}$ tradução poética, predominam os elementos materiais do signo e a passagem para um nivel superior de significação oblíqua, o nivel semiótico da significância, o que permite a leitura múltipla pela quebra da referencialidade externa.

Il ne s'agit pas de discuter ici la question de savoir si la poésie est ou non traduisible. Partons du fait que la poésie a toujours été traduite et qu'elle le sera toujours, et examinons plutôt la spécificité de l'opération traduisante qui a pour objet la poésie dans sa manifestation textuelle la plus caractéristique : le poème.

Remarquons d'abord qu'on peut traduire de la poésie sans faire pour autant de la traduction poétique, comme le font, trop souvent hélas, nombre de traducteurs, surtout en France. Nous allons justement essayer de repérer dans le texte ce qui constitue la manifestation du «poétique» et de vérifier quelles opérations, dans l'acte de traduction, permettent de «faire passer» ce poétique dans le texte traduit; autrement dit, comment traduire un texte de type poétique de sorte qu'il soit rendu comme poésie dans la langueculture d'accueil.

Dans la distribution typologique des textes, il n'y a pas de compartiments étanches, cloisonnés, mais plutôt deux pôles vers lesquels tendent les textes, occupant des espaces aux limites plus ou moins indéfinies.

D'un côté se trouve le pôle du concept, du signifié, et à l'autre extrême, le pôle du signifiant, de l'élément matériel du signe. Certains textes sont marqués par la primauté du concept. Ce qu'il est important de rendre, dans ce cas, ce sont les idées, les relations, les 
faits, les procès. Il y a compromis avec une réalité extérieure au texte, avec une rationalité considérée comme objective, avec une logique qui se règle par le critère de vérité. Dans ce cas, le rapport entre le signifié et le signifiant apparaît comme tout à fait arbitraire, et le caractère linéaire du signifiant s'impose. Celui-ci est réduit à la condition de véhicule du concept, ce qui a fait appeler «véhiculaire» ce premier type de textes. Dans cette catégorie se rangent les textes dits scientifiques, techniques, informatifs, pragmatiques, argumentatifs, etc. Ainsi, dans le rapport d'une expérience physique ou d'une opération chirurgicale, dans la démonstration d'un théorème de géométrie, dans le mode d'emploi d'un appareil, dans le récit d'un accident de la route, la primauté du concept sur la matérialité du signe s'impose, et le rapport avec un référent extérieur nécessite une lecture linéaire et univoque pour que soit pleinement accomplie la fonction du texte.

Le traducteur, donc, quand il réćcrit un texte de ce type, doit veiller en premier lieu à ce que le sens soit respecté le plus étroitement possible; pour cela, le choix du lexique et de la structure des phrases doit être fait en fonction des concepts véhiculés de façon à permettre cette lecture univoque et claire qui évitera des «fautes d'interprétation» et assurera l'équivalence du texte d'arrivée au texte de départ en ce qui a trait à sa performance du point de vue de la fonction communicative.

D'autre part, il y a les textes tendant vers le pôle du signifiant, où la matérialité du signe prévaut sur le concept : le phonème récupère sa valeur en tant que «son», l'écriture prend souvent des aspects icôniques, l'arbitraire du signe s'affaiblit au profit de la motivation des rapports entre signifié et signifiant, et celui-ci cesse d'être simple véhicule du premier pour en venir à le déterminer, à l'engendrer. La lecture linéaire est remplacée par la lecture rétroactive et tabulaire qui redéfinit les rapports entre les éléments de la chaîne discursive. Envisagée du point de vue non simplement linguistique mais sémanalytique, la linéarité syntaxique est battue en brèche, laissant filtrer le sémiotique dans le symbolique (cf. Kristeva 1974: 40, 67-69 et passim) sous l'action des pulsions corporelles du sujet. C'est ce nouveau mode de production du sens qui a lieu à l'intérieur du texte dans le jeu des forces que sous-tendent les signifiants qu'on appelle la signifiance, par opposition au sens référentiel.

La signifiance est responsable de l'ouverture de la signification à des lectures multiples, toutes plausibles, et c'est là l'une des marques du texte poétique, par opposition à l'univocité du texte véhiculaire. On le voit, nous sommes là devant une tout autre façon de signifier, qui est la marque du texte poétique, du poème. Selon Michael Riffaterre «le poème nous dit une chose et en signifie une autre et cela s'explique entièrement $\mathrm{p}_{\mathfrak{a}}$ la façon dont le texte poétique génère son sens» (Riffaterre 1983: 11). Ce même aute . (p. 13 et suivantes) réserve le terme de sens pour l'information fournie par le texte mimétique $^{1}$ et emploie le terme signifiance pour désigner cette «unité formelle et sémantique qui contient tous les indices de l'obliquité». Ces indices signalent que le texte fait éclater les limites de la mimésis et qu'il doit être lu et interprété dans une autre dimension, celle de la sémiosis, qui dépasse le niveau strictement linguistique. La signifiance est une manifestation de la sémiosis.

Le traducteur du poème doit donc avoir devant son texte une attitude bien différente de celle du traducteur d'un texte véhiculaire. Tandis que celui-ci traduit surtout le sens, le premier doit, dans son opération de réécriture, faire passer dans son texte la signifiance spécifique du poème original, car elle en constitue la carte d'identité.

Mais comme l'activité du traducteur débute toujours par une lecture, il faut que ce lecteur-scripteur tienne compte de tous ces faits observables et identifiables comme responsables de l'obliquité sémantique du poème, i.e. les marques textuelles de la signifiance, et qu'il travaille à les récupérer dans le texte qu'il produit. Ces marques sont nombreuses. J'en indiquerai quelques-unes parmi les plus importantes. 
Pour outrepasser les limites de la mimésis et atteindre la signifiance, le lecteurtraducteur doit vaincre certains obstacles dont le premier est constitué par les agrammaticalités. Si l'on admet que la grammaire est le socle qui soutient le pilier de la signification référentielle, toute violence à la normalité grammaticale peut être vue comme une dérogation de la mimésis, c'est-à-dire que les agrammaticalités sont des indices que le texte doit être lu à un autre niveau. Le sens, menacé au niveau mimétique, se recompose au niveau supérieur de la sémiosis. Ainsi, les agrammaticalités, qui ne suffisent pas à elles seules à constituer le niveau sémiotique du texte, doivent cependant être considérées comme des clés de la signifiance.

Je rappelle que le terme d'agrammaticalité est employé ici dans un sens large et non exclusif, contrairement à l'usage qu'en font les grammairiens générativistes. Il peut désigner depuis les cas minimaux de perturbation de la linéarité syntaxique jusqu'aux cas extrêmes qui conduisent à l'hermétisme ou frôlent le non-sens. Le vers lui-même, qu'il soit régulier ou libre, peut être vu comme une sorte d'agrammaticalité dans la mesure où il se définit comme perturbation de la linéarité grammaticale:

La mise en ordre syntaxique des éléments dans la phrase et de phrase en phrase se trouve, en poésie, travaillée et contestée par le vers. À la différence de la prose comme «discours qui va de l'avant», le vers déplace les éléments et superpose les principes du mètre et du parallélisme à la linéarité grammaticale. (Adam 1985:221)

De même, les récurrences lexicales, sémantiques, syntaxiques et phoniques qui attirent 1'attention du lecteur par leur caractère insolite peuvent être considérées comme des agrammaticalités lato sensu.

Le traducteur, qui doit «traduire les agrammaticalités» et non pas les éliminer, sous peine de perdre l'une des clés de la signifiance, sera souvent embarrassé du fait que des langues différentes ont des grammaires différentes et, en conséquence, les agrammaticalités ne sont pas les mêmes, ou ne sont pas de même nature. Ainsi, l'antéposition de l'adjectif peut, dans certains cas, constituer une agrammaticalité en français, en portugais et dans les langues romanes en général. Cette agrammaticalité ne peut pas être traduite par une agrammaticalité de même nature en anglais du simple fait que, dans cette langue, l'antéposition de l'adjectif constitue la règle. Le traducteur doit donc violer un autre point de la grammaire pour récupérer un niveau équivalent d'agrammaticalité.

Un autre indice textuel de la signifiance qu'il est important de signaler est le signe double. Michael Riffaterre définit le signe double ou point nodal comme «un mot équivoque situé à l'intersection de deux séquences d'associations sémantiques ou formelles» (1983: 113). Quand le signe double se trouve être le titre, sa force comme facteur d'obliquité en est extraordinairement accrue, car le titre est souvent la matrice dont le poème est l'expansion. Comme le signe double est en réalité un signifiant unique qui, dans une langue donnée, vehicule deux signifiés différents, son utilisation en poésie constitue un cas particulier de «jeu de mots». Or, chacun sait que le jeu de mots est en général intraduisible en raison du fait que dans une autre langue on aura normalement un signifiant différent pour chacun des siginifiés du signe double. Le traducteur de poésie doit faire valoir son imagination et son domaine des ressources de sa langue pour récupérer l'effet de poésie qui, dans l'original, découle de l'emploi d'un signe double, sinon, un élément de la signifiance sera irrémédiablement perdu. C'est le cas de la traduction du poème de Carlos Drummond de Andrade, intitulé Poema-orelha, par Jean-Michel Massa (1973: 120-121), dont je transcris le titre et les quatre premiers vers: 


\author{
POÈME-OREILLE \\ Voici l' oreille du livre \\ par où le poète écoute \\ si on parle mal de lui \\ ou si on l'aime.
}

C'est une traduction mot à mot de l'original brésilien. Mais Drummond avait joué avec le mot orelha qui en portugais est un signe double; il désigne soit le pavillon extérieur de l'appareil auditif, soit le repli de la couverture d'un livre sur lequel l'éditeur fait souvent insérer des commentaires sur le contenu du volume. Une telle ambiguïté n'existant pas en français, l'obliquité de signification est perdue. Voici l'oreille du livre est perçu en français comme une figure d'invention, comme une métaphore nouvelle, tandis qu'en portugais orelha do livro est une lexie d'usage courant attestée par les dictionnaires. L'intérêt et l'originalité du texte brésilien réside dans le fait que le poème est l'expansion du titre-matrice, cela veut dire que la grammaire de la signifiance du texte consiste justement à exploiter les rapports existant entre les deux signifiés que recouvre un seul et même signifiant.

Les interprétants textuels constituent un troisième indice du passage du niveau mimétique au niveau sémiotique ou de la signifiance dont la traduction poétique doit tenir compte. «Le passage du sens à la signifiance impose le concept d'interprétant, un signe qui gouverne l'interprétation des signes surfaciels du texte et explicite tout ce que ces signes ne font que suggérer», nous dit encore Riffaterre ${ }^{2}$ (1983: 107).

Examinons, à titre d'exemple, le court poème de Jacques Prévert (1985: 38-39):

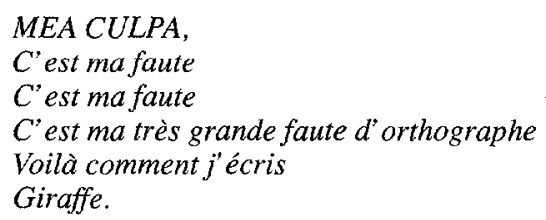

Le titre du poème est un interprétant textuel dans la mesure où il renvoie au confiteor, prière qui intègre la tradition chrétienne et qui, dans chaque langue-culture, possède une formule canonique, officielle, une forme figée. Prévert reproduit ipsis litteris une partie du texte canonique en français: «C'est ma faute, c'est ma faute, c'est ma très grande faute». Nous sommes immergés, par cet interprétant, dans l'isotopie du rituel chrétien, du péché et du pardon... Mais, à la grande surprise du lecteur, au beau milieu du poème, il y a une rupture provoquée par le signe double faute qui, tout en appartenant à l'isotopie de la religion, par son déterminant «d'orthographe» nous lance dans l'isotopie de «l'école», où se situe toute la suite du texte. Le mode d'expansion de la matrice ou la grammaire de la signifiance est donc axé sur un interprétant textuel (confiteor) et sur un signe double (faute). Santiago:

Voici la traduction de ce poème faite par le poète et traducteur brésilien Silviano

\author{
MEA CULPA \\ Errei \\ Errei \\ Que enorme erro de ortografia \\ Eis como escrevi \\ Girrafa.
}

Le texte traduit a entièrement perdu la poéticité spécifique de l'original pour la simple raison que le traducteur n'a pas su garder la grammaire de la signifiance, c'est-à-dire 
que dans le texte d'arrivée, l'interprétant textuel, qui serait le texte canonique du confiteor en portugais ( «Minha culpa, minha culpa, minha máxima culpa...») et le signe double disparaissent.

Je proposerais donc la traduction suivante, qui prend en ligne de compte les observations ci-dessus :

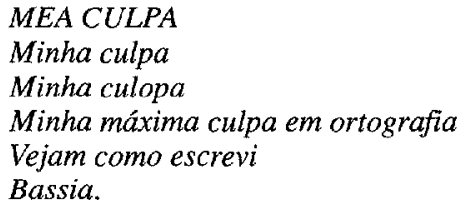

Ainsi, en plus de garder l'interprétant et le passage de l'isotopie de la «religion» à l'isotopie de «l'école», on a pu préserver quelques autres éléments de la signifiance comme le rythme de l'original et la primauté de l'élément matériel du signe sur le concept. Sans aucun doute, en traduisant «giraffe» («girrafa» dans la traduction de S. Santiago) par «bassia» («cuvette» en français), j'ai fait une opération tout à fait inconcevable en traduction pragmatique, mais parfaitement plausible en traduction poétique. En effet, quand Prévert a choisi le mot «giraffe» pour boucler son texte, il ne l'a pas fait en fonction de ses composantes conceptuelles (animal mammifère, quadrupède, au long cou, etc.), mais pour deux raisons spécifiques: d'abord pour la récurrence phonique (orthographe/giraffe) et ensuite parce que le redoublement du «f» dans ce mot reste dans le domaine de l'orthographe, sans aucune conséquence phonétique ou phonémique. Il en est de même pour le mot «bassia» (dont l'orthographe correcte en portugais est «bacia»), qui rime avec «ortografia» et contient une faute strictement orthographique. Seuls les éléments matériels du mot ont été considérés. Or, l'option de S. Santiago, en essayant de garder les éléments conceptuels de «girafe» («girafa» en portugais) a perdu la récurrence phonique et a ajouté une faute de phonétique à la faute d'orthographe: en portugais «rr» se prononcent différemment de «r».

D'autres marques textuelles de la signifiance mériteraient d'être examinées ici en vue de la traduction "poétique» du poème, mais les limites d'un article imposent des restrictions. Je me bornerai donc à en citer encore une pour terminer. Il s'agit de ce que J.-M. Adam (1985: 29), reprenant un article de Jacques Anis, appelle la vi-lisibilité du texte poétique. Nous avons vu qu'on accède à la signifiance du poème par une lecture à deux étapes: la première, linéaire, mimétique; la seconde, rétroactive, tabulaire, sémiotique. Il y a cependant une sorte de pré-lecture strictement visuelle, basée sur la distribution spatiale de la masse textuelle sur la page. Elle ne participe pas de la discursivité linguistique, mais se présente plutôt comme une perception globale et achronique, non séquentielle, comme dans les arts plastiques.

Voir dans le poétique un art de la parole, c'est situer le message dans le temps et ainsi privilégier le décodage séquentiel qui institue une hiérarchie linêaire des matériaux linguistiques à prendre en considération dans un certain ordre. Prendre au contraire la page comme lieu de la manifestation poétique, c'est donner d'emblée la première place à un décodage globalisant [...]. Le poème apparaîtra d'abord comme un macro-signe spatialisé... (Delas et Filliolet 1973: 176)

La vi-lisibilité a comme fonction primordiale d'engendrer l'effet de poésie ou «l'effet-poème» (cf. Adam 1985: 29). En jetant le regard sur la page où s'insère le texte, on voit qu'il s'agit d'un poème et non d'un article de journal, d'une lettre ou d'un conte, et cela crée chez le lecteur la prédisposition à une lecture poétique, non référentielle, qui recherchera le sens oblique, la signifiance poétique. 
Pour le traducteur de poèmes, la traduction commence par la transposition de la vi-lisibilité. Un sonnet doit être traduit par un sonnet, un poème en vers libres par un poème en vers libres et ainsi de suite. Procéder autrement serait s'éloigner de la traduction vers la re-création libre ou, pour reprendre le vieux mot de Joachim du Bellay, vers la simple innutrition. La traduction, elle, a un compromis avec la vi-lisibilité de l'original. Ce compromis admet une certaine flexibilité, bien sûr, mais le traducteur ne peut pas l'ignorer et doit toujours essayer de la récupérer. Le respect de la vi-lisibilité joue un rôle important dans la traduction des poèmes de toutes les époques dans la mesure où elle est la gardienne de certains traits qui situent les textes dans leur temps et dans leur espace culturel; mais cette importance est accrue quand il s'agit des textes de certains poètes modernes comme e. e. cummings ${ }^{3}$, ou les concrétistes, par exemple.

En somme, si l'on peut parler de fídélité en traduction, la fidélité en traduction poétique consistera dans la récupération, dans le texte d'arrivée, des marques textuelles de la signifiance, de sorte que le texte d'arrivée puisse être non seulement un poème dans la langue-culture d'accueil, mais un poème homogène par rapport au poème original dans ce qui constitue son identité poétique.

\section{Notes}

1. «La mimésis est caractérisée par une séquence sémantique à variation continue, cela parce que la représentation est fondée sur le caractère référentiel de la langue» (Riffaterre 1983: 13).

2. Comme on le voit, la conception d'interprétant chez Riffaterre a une extension plus large que chez Peirce (cf. Peirce 1977: 43).

3. Sur le rôle de la spatialité dans la traduction de e. e. cummings, voir l'article de Guy Leclerc «Traduire de la poésie, c'est faire de la poésie. Quelques jalons dans l'approche d'un poème de e. e. cummings», Revue d'esthétique, $\mathbf{n}^{\circ} 12$ (Spécial traduction), Toulouse, Privat, 1987, pp. 107-119.

\section{RÉFÉRENCES}

ADAM, J.-M. (1985) : Pour lire le poème, Bruxelles, A. De Boeck.

DELAS, Daniel et Jacques FILLIOLET (1973) : Linguistique et poétique, Paris, Larousse.

DRUMMOND DE ANDRADE, Carlos (1973) : RéunioniReunião, poèmes choisis, traduits et préfacés par JeanMichel Massa, Paris, Aubier Montaigne.

KRISTEVA, Julia (1974): La révolution du langage poétique, Paris, Seuil

LECLERC, Guy (1987): «Traduire la poésie, c'est faire de la poésie», Revue d'esthétique, $\mathrm{n}^{\circ} 12, \mathrm{pp} .107-119$.

PEIRCE, Charles S. (1977) : Semiótica, São Paulo, Perspectiva.

PRÉVERT, Jacques (1985): Poemas, seleçäo e tradução de Silviano Santiago, Rio de Janeiro, Nova Franteira.

RIFFATERRE, Michael (1983): Sémiotique de la poésie, Paris, Seuil. 